\title{
Evodiamine inhibits human thyroid cancer cells in vitro and in vivo
}

\section{Ying-Ray Lee1, Chieh-Hsiang Lu², Yi-Ping Chang1, Yi-Sheng Chang ${ }^{1}$, Shu-Hsin Chen ${ }^{1}$, Hui-I Yu²}

1 Department of Medical Research, Chiayi Christian Hospital, Chiayi, Taiwan

2 Division of Endocrinology and Metabolism, Department of Internal Medicine, Chiayi Christian Hospital, Chiayi, Taiwan

\section{Introduction:}

Thyroid cancer is the most prevalent cancer among endocrine malignancies. In clinically, surgical resection combined with radioactive iodine therapy has been proved effective in treating differentiated thyroid cancer, including papillary and follicular thyroid cancers (FTC). However, patients with incurable differentiated thyroid cancer (DTC), poorly differentiated thyroid cancer (PDTC) and anaplastic thyroid cancer (ATC) exhibit worse prognosis. Therefore, a novel and effective treatment is urgently needed to deal with the current treatment.

Evodiamine is one of the important components isolated from Chinese herb Wu-Chu-Yu, and has been demonstrated to contribute on anti-inflammatory effects, anti-angiogenesis, anti-tumor growth, anti-invasive and metastatic activities, and up-regulating apoptosis. In the present study, we examined the effects of evodiamine in FTC, PDTC and ATC cells, respectively. Evodiamine inhibited cellular proliferation and the colonies formation of FTC, PDTC and ATC cells. Cell cycle arrest at G2/M phase was found in all of the cells during evodiamine treatment. Moreover, caspase-dependent apoptosis in these cells was also revealed under evodiamine treatment. In addition, autophagy induction was also found in evodiamine treated human thyroid cancer cells. Finally, we verified the effects of evodiamine on anti-human thyroid cancers in a xenograft nude mice model. Our results demonstrate that evodiamine induces cell cycle arrest, caspase-dependent apoptosis and autophagy leading to inhibit proliferation of multiple types of human thyroid cancer cells. We suggest that evodiamine could be a chemo-therapeutic candidate for human thyroid cancers.

\section{Results:}

I. Evodiamine suppressed cell proliferation in ARO, WRO and SW579 cells

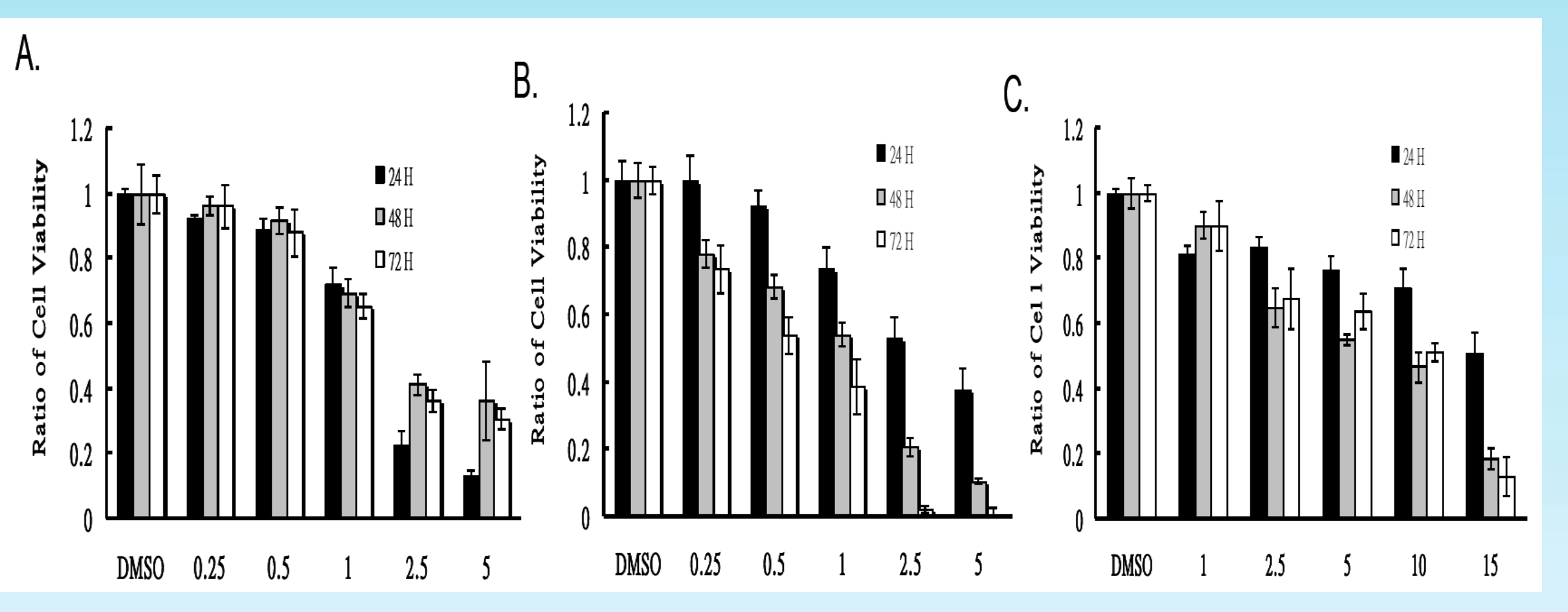

Figure 1. Viability of human thyroid cancer cells treated with or without evodiamine. (A) ARO, (B) SW579 and (C) WRO cells were treated with DMSO or evodiamine with various dosages and the cell viability was detected with CCK-8 assay. The results were expressed as mean \pm SEM of three independent experiments.

II. Evodiamine inhibited colonies formation in ARO cells

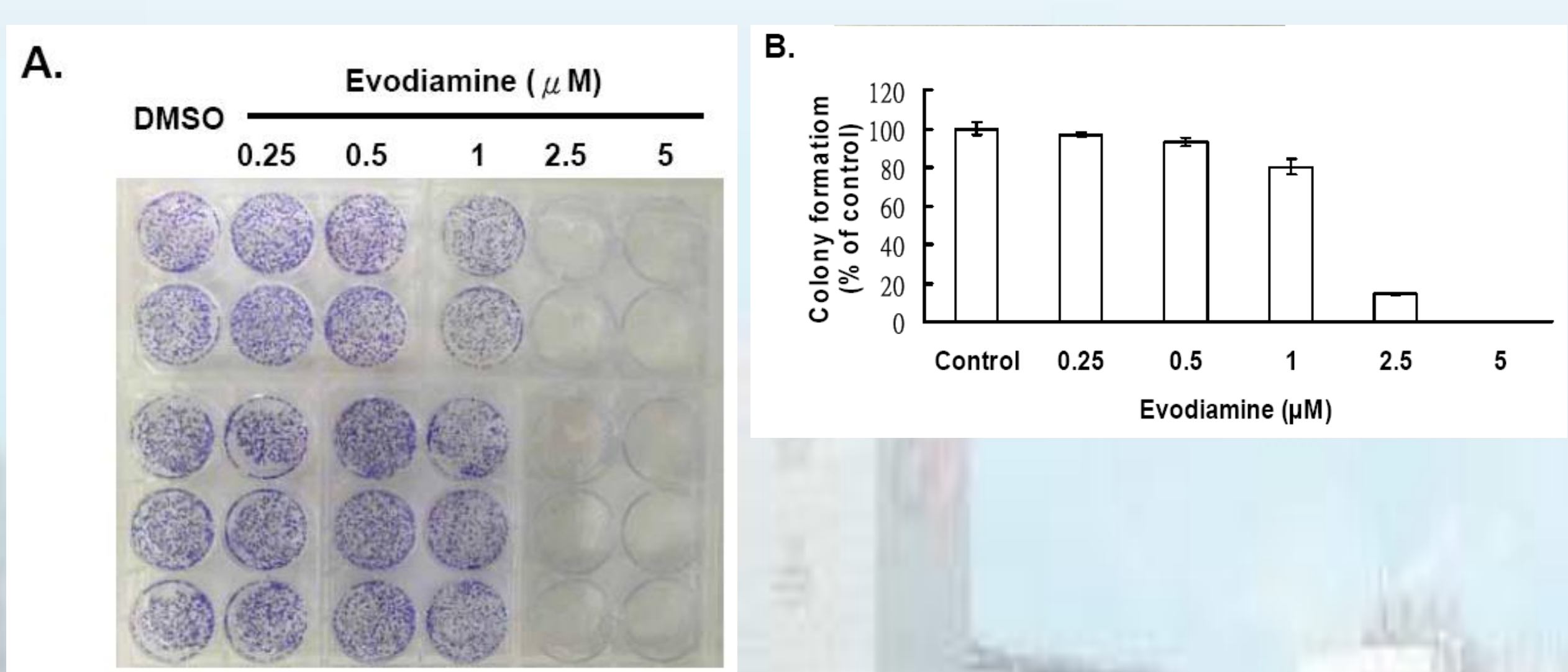

Figure 2. Colony formation of human thyroid cancer cells under evodiamine treatment. (A) ARO cells were treated with or without evodiamine for 14 days, and the colony formation were determined. (B) the numbers of tumor-colonies were counted. The DMSO was used as a negative control. The results were expressed as mean $\pm S E M$ of three independent experiments.

\section{Evodiamine induced G2/M arrest}

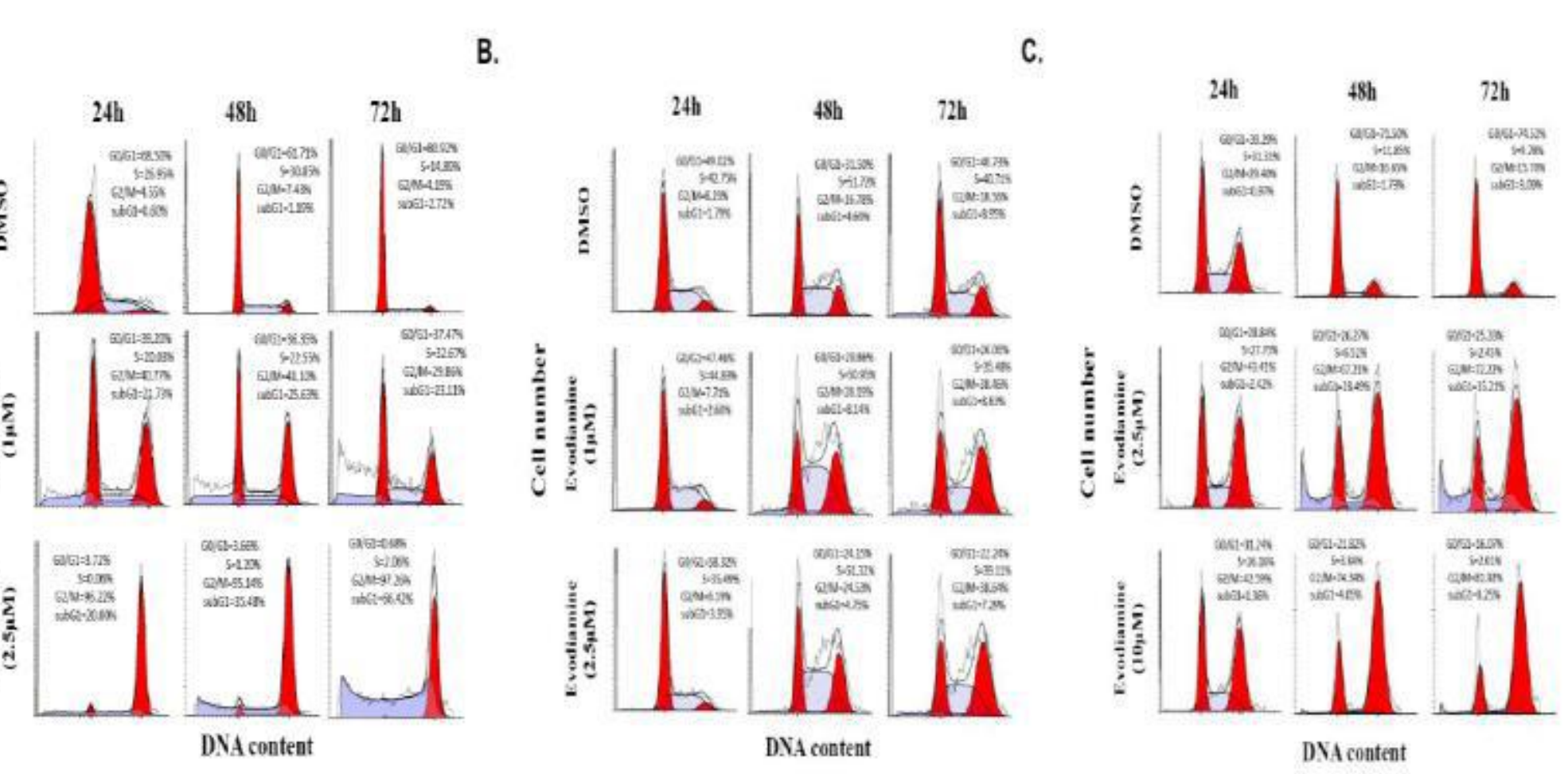

Figure 3. Evodiamine treatment induced cell cycle arrest in human thyroid cancer cells. Human thyroid cancer cells (A) ARO (B) SW579 and (C) WRO were treated without or with evodiamine, and the cell cycle was determined by flow cytometry after various post-treatment. DMSO was used as a negative control.

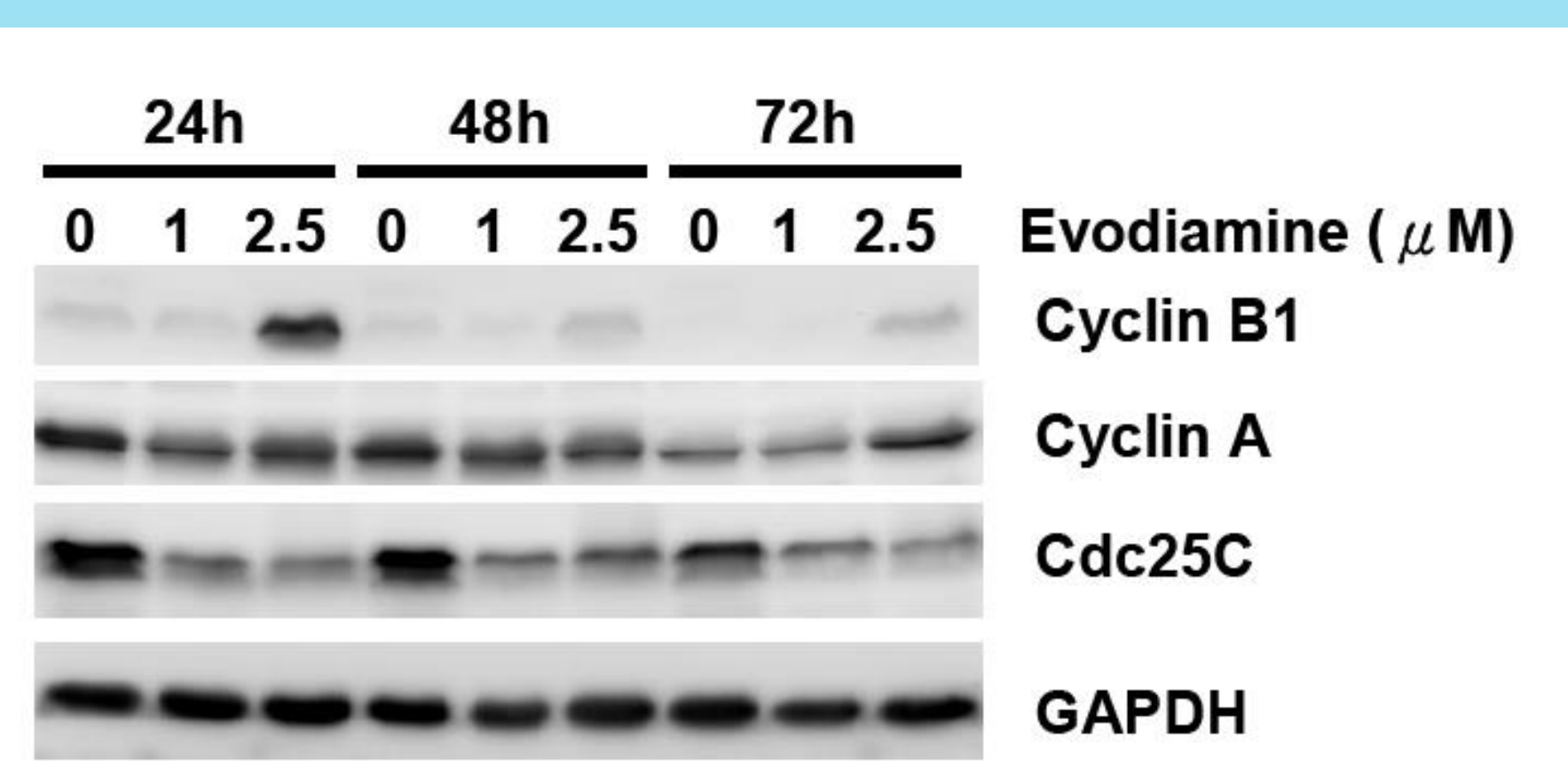

Figure 4. Evodiamine altered the expressions of cell cycle-related proteins. ARO cells were treated with evodiamine, and the expressions of cyclin $A$, cdc25c and cyclin B1 were measured by Western blot analysis. The amount of GAPDH was measured as an internal control.

\section{Evodiamine induced apoptosis in} WRO cells

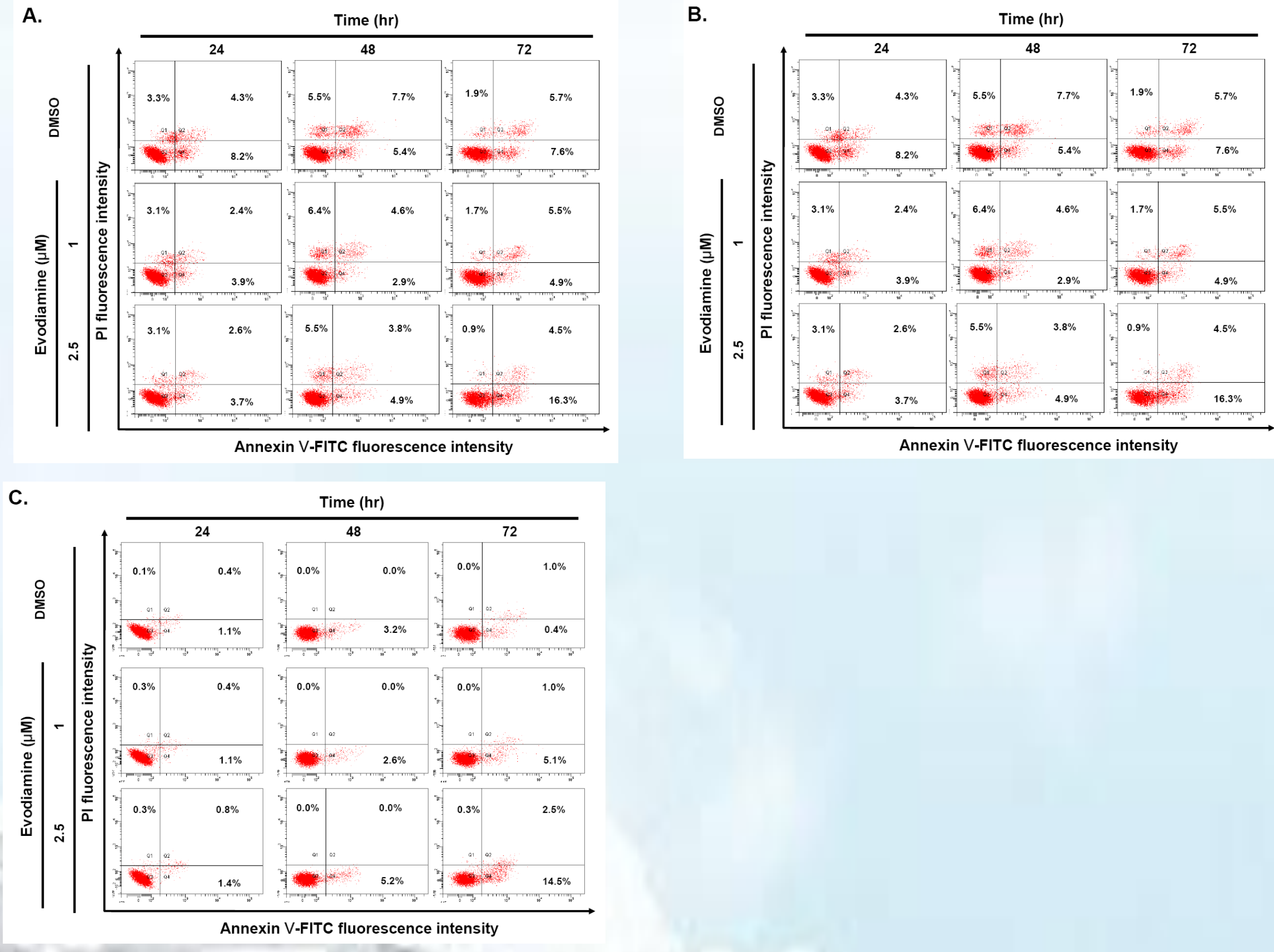

Figure 5. Apoptosis induction in evodiamine treated human thyroid cancer cells. (A) ARO, (B) WRO and (C) SW579 cells were treated with evodiamine and the cellular apoptosis was determined by flow cytometry. DMSO was used as the control group.

V. Evodiamine elevated caspasedependent apoptosis

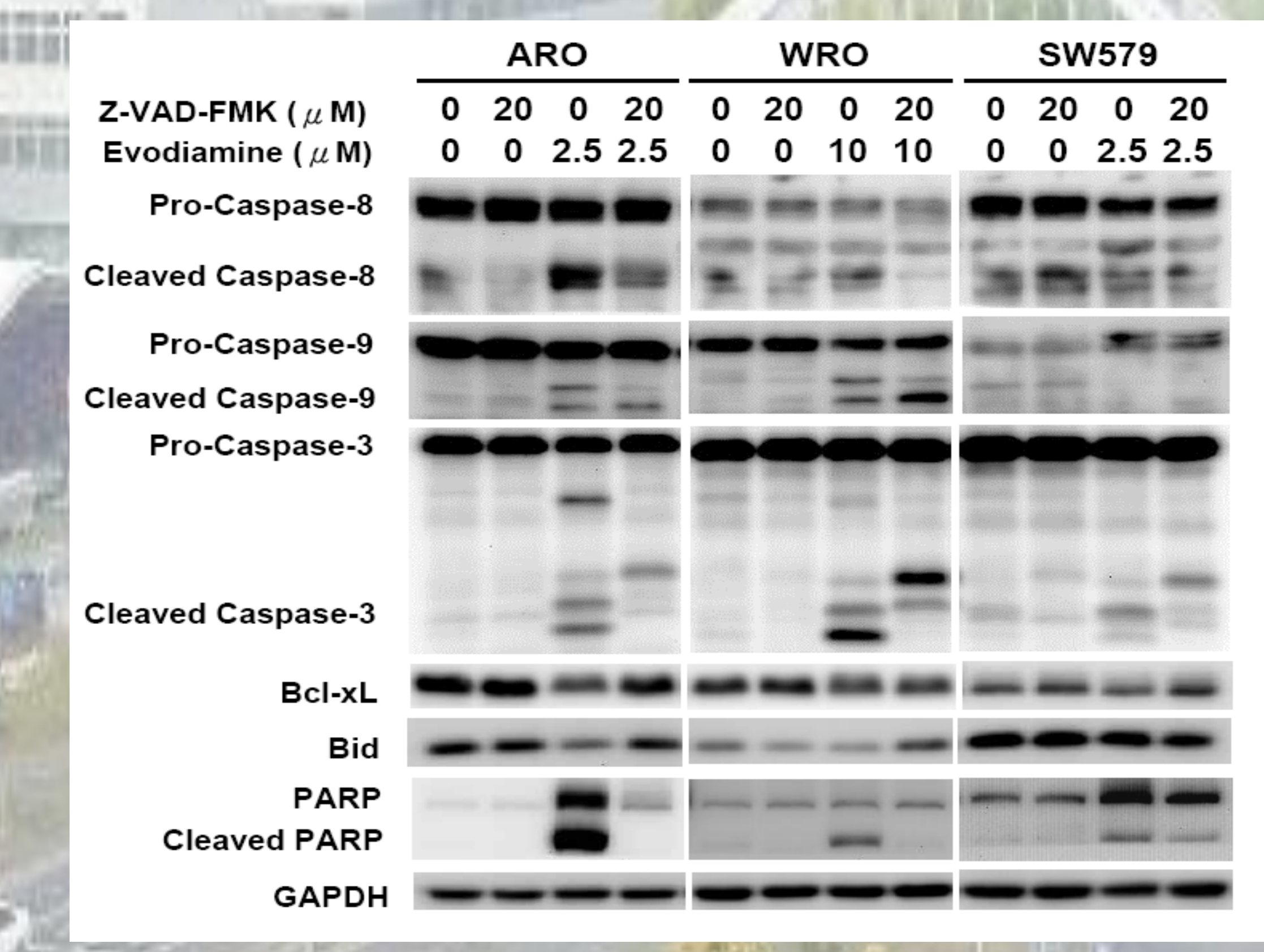

Figure 6. Evodiamine induced caspase-dependent apoptosis, and apoptosis occurred through an extrinsic and/or intrinsic pathway in human thyroid cancer cells. ARO cells and SW579 were incubated with evodiamine for $72 \mathrm{hrs}$ and WRO cells were treated with evodiamine for $24 \mathrm{hrs}$ and the expressions of caspase, Bid, Bcl-XL and PARP were detected by Western blotting. Z-VAD-FMK, a pan-caspase inhibitor, was used to block the activations of caspase mediated by evodiamine and GAPDH was used as a loading control.

VI. Evodiamine induced autophagy in ARO and WRO cells

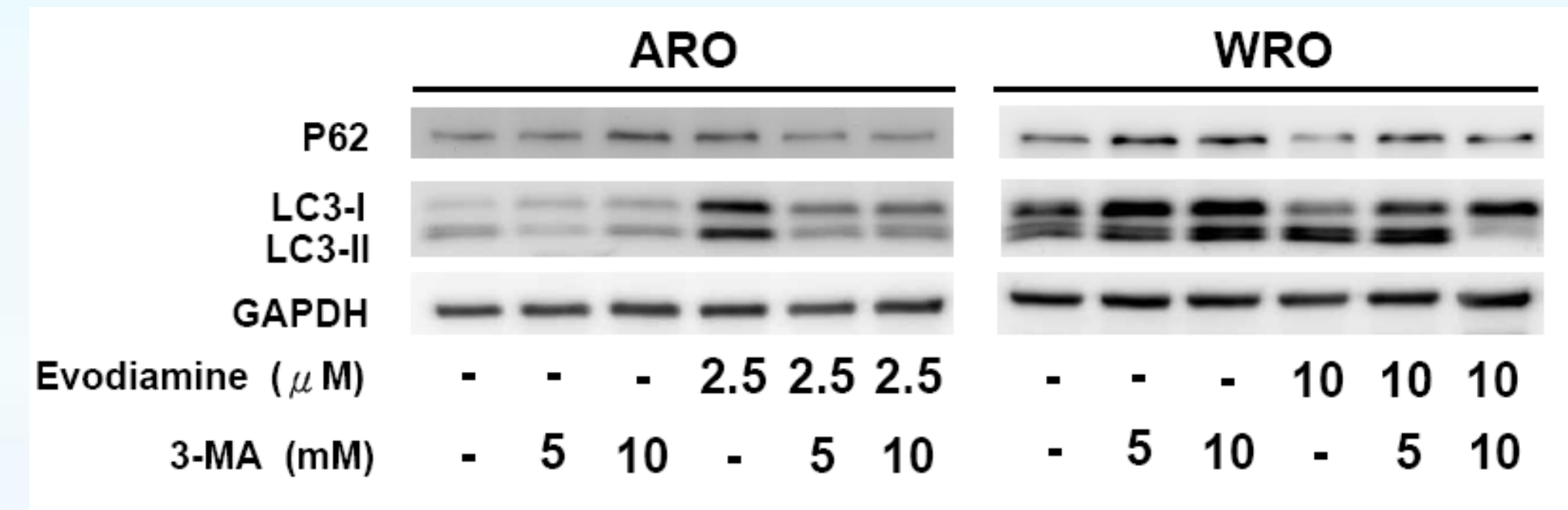

Figure 7. Evodiamine induced autophagy in human thyroid cancer cells. ARO and WRO cells were treated with evodiamine for 72 and $24 \mathrm{hrs}$, and the expressions of LC3 and p62 were determined by Western blotting. GAPDH was used as a loading control, and DMSO was used as a negative control.

\section{Evodiamine suppressed tumorigenesis of human thyroid cancer cells in a xenograft nude mice model}

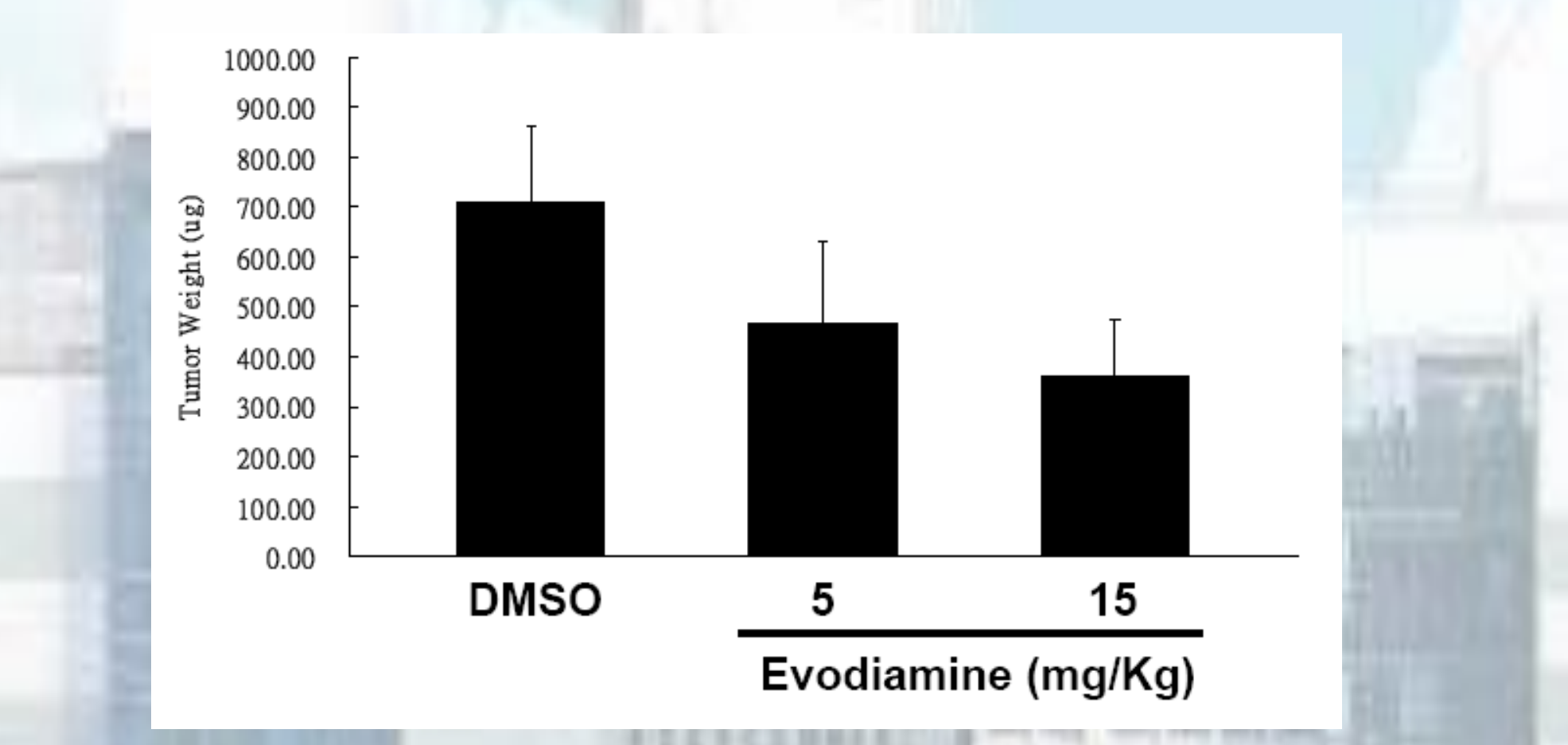

Figure 8. Evodiamine inhibited tumorigenesis of human thyroid cancer cells in a nude mice model. The mice were injected subcutaneously with ARO cells, and treated without or with evodiamine, the tumor weight were determined after 21 days treatment.

\section{Conclusions:}

In the present study, we demonstrated that evodiamine could suppress human thyroid cancer cells proliferation Cell cycle arrest, caspase-dependent apoptosis and autophagy were observed in human thyroid cancer cells during evodiamine treatment. Moreover, evodiamine was demonstrated to suppress the tumorigenesis in vitro and in vivo. Our findings elevate that evodiamine is a potential therapeutic agent against incurable human thyroid cancer and is worthy of further clinical investigation. 